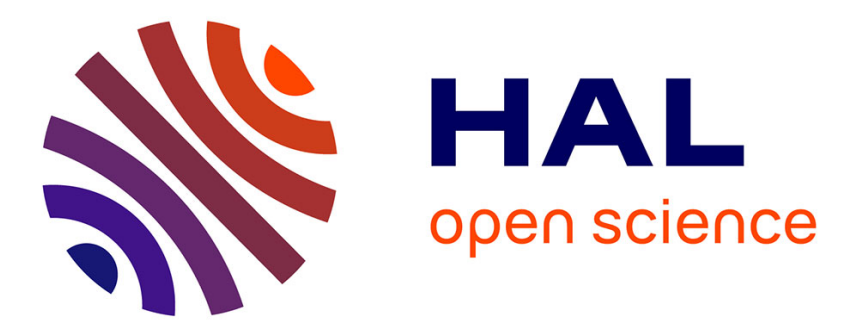

\title{
Structural considerations and cognitive complexity in air traffic control
}

Jonathan Histon, John Hansman, Blake Gottlieb, Howard Kleinwaks, Sarah Yenson, Daniel Delahaye, Stéphane Puechmorel

\section{To cite this version:}

Jonathan Histon, John Hansman, Blake Gottlieb, Howard Kleinwaks, Sarah Yenson, et al.. Structural considerations and cognitive complexity in air traffic control. DASC 2002, 21st Digital Avionics Systems Conference, Dec 2002, Irvine, United States. pp 1C2-1 - 1C2-13 vol.1, 10.1109/DASC.2002.1067894 • hal-00938407

\section{HAL Id: hal-00938407 \\ https://hal-enac.archives-ouvertes.fr/hal-00938407}

Submitted on 5 May 2014

HAL is a multi-disciplinary open access archive for the deposit and dissemination of scientific research documents, whether they are published or not. The documents may come from teaching and research institutions in France or abroad, or from public or private research centers.
L'archive ouverte pluridisciplinaire HAL, est destinée au dépôt et à la diffusion de documents scientifiques de niveau recherche, publiés ou non, émanant des établissements d'enseignement et de recherche français ou étrangers, des laboratoires publics ou privés. 


\title{
STRUCTURAL CONSIDERATIONS AND COGNITIVE COMPLEXITY IN AIR TRAFFIC CONTROL
}

\author{
Jonathan M. Histon, R. John Hansman, Blake Gottlieb, Howard Kleinwaks, Sarah Yenson, MIT, \\ Cambridge, $M A$
}

Daniel Delahaye, Stephane Puechmorel, ENAC / CENA, Toulouse, France

\begin{abstract}
The ability of air traffic controllers to deal with complex situations is a limiting factor in airspace capacity. The underlying airspace structure and other procedural elements are thought to be important factors in reducing a controller's Cognitive Complexity through the use of structure based abstractions. Because Cognitive Complexity cannot be directly observed it must be investigated indirectly. This paper discusses and presents examples of how directly observable states and controllers subjective responses can be used to indirectly probe and gain insight into how structure based abstractions are used to manage Cognitive Complexity.
\end{abstract}

\section{Introduction}

The Cognitive Complexity of an air traffic situation is an important factor in determining airspace capacity. Cognitive Complexity is generally assumed to correlate with the cognitive difficulty of controlling the air traffic situation under normal and abnormal conditions. The objective of the work discussed in this paper is to understand the factors that influence complexity, particularly those factors which relate to the underlying structural elements in air traffic control.

A better understanding of all of the factors that impact Cognitive Complexity is important for advancing both safety and efficiency in the ATC system. A controller can become overloaded if the Cognitive Complexity of a situation exceeds their capabilities. In current operations, traffic management procedures restrict capacity to prevent such situations from developing.

Because structure can be imposed by external factors, understanding the role of structure can help assess the potential impact of proposed changes to the ATC system such as airspace redesign, modifications of operational procedures or the introduction of new technologies.

Previous metrics of ATC complexity have concentrated on geometrical factors [1] [2] [3] [4]. A deeper understanding of how structure impacts Cognitive Complexity can extend these previous efforts and help provide a more complete understanding of the critical system properties for operational and design applications.

\section{Structure and Cognitive Complexity}

In order to understand how structure influences a controllers Cognitive Complexity a simple model is presented to provide a framework linking structure based abstraction with the controllers mental model and the air traffic control task.

\section{Generalized ATC Process Model}

Figure 1 presents a simple model which depicts hypothesized relationships between structure, Cognitive Complexity, and the basic tasks of air traffic control. In the model the controller receives surveillance information about the air traffic situation and processes it to produce a set of output commands [5]. The processing of the information is represented in terms of an adapted version of Endsley's model of Situation Awareness [6]. 


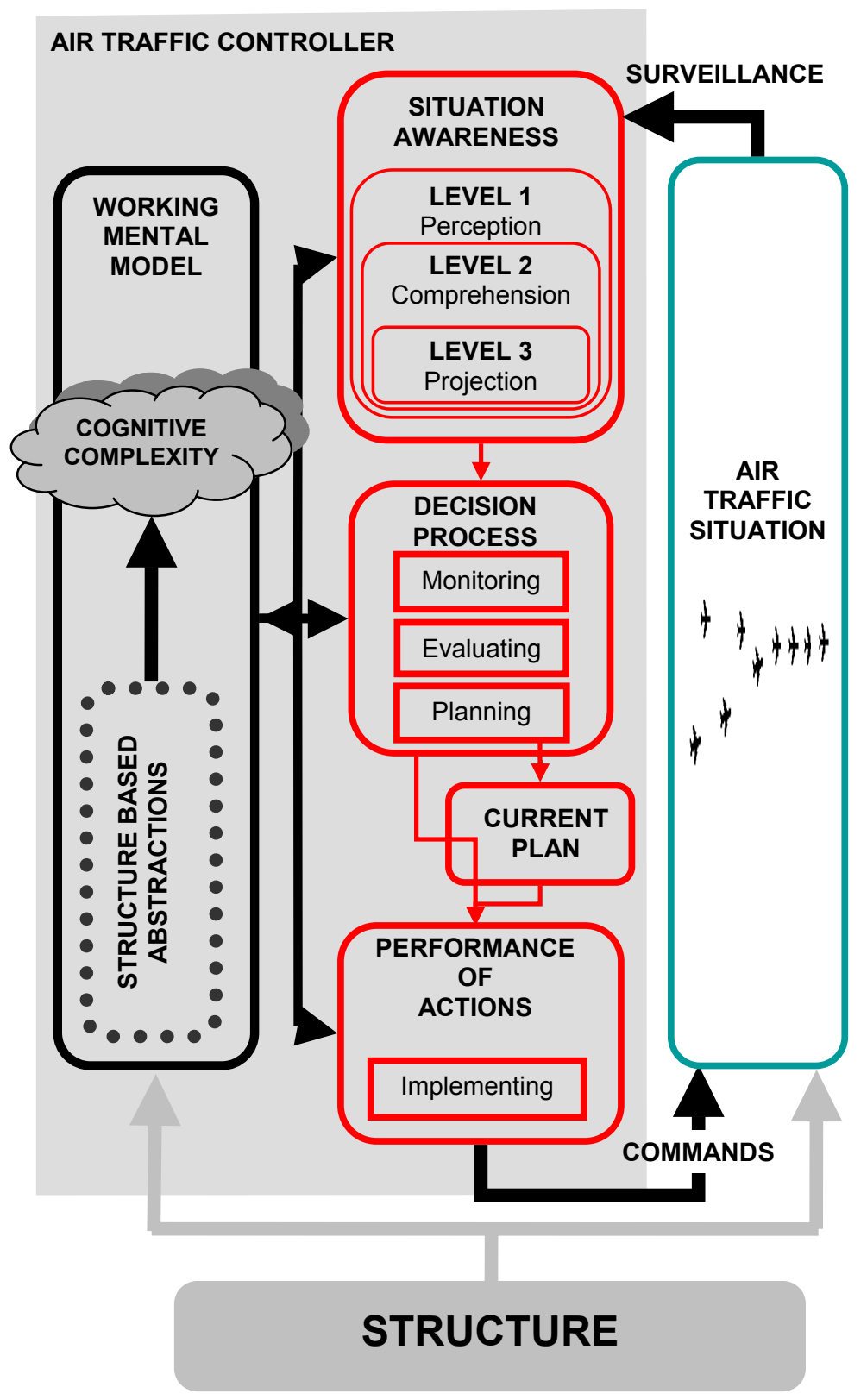

Figure 1. Generalized ATC Process Model

Key high-level ATC processes of planning, evaluating, and monitoring, identified by Pawlak et al., are included as parts of the decision making process [7]. The planning process produces a distinct cognitive element identified in the model as the controller's "Current Plan" [5] which is an internal representation of the schedule of events and commands to be implemented as well as the resulting aircraft trajectories that will ensure that the air traffic situation evolves in an efficient and conflict-free manner.
In Figure 1, Cognitive Complexity is associated with the working mental model of the controller. It is hypothesized that the needs of the controller's current task will determine the working mental used to accomplish that task. The working mental model is an internal representation of the dynamics of the real system and will operate at a level of abstraction appropriate for the task being performed. Cognitive Complexity is an attribute of the controllers working mental model and is related to the scope and details of the factors being 
considered as well as any simplifying abstractions employed by the controller.

In Figure 1, structure is presumed to be a basis for complexity reducing abstractions. Structure is composed of elements that shape and influence the evolution of the air traffic situation including physical objects, such as radio beacons (e.g. VORs, NDBs etc...), and non-physical elements such as rules, procedures, and constraints. Structure can also be an internal construct such as techniques or standard procedures used in a given situation. Such internalised structures are difficult to identify and capture but appear to be important mechanisms by which controllers can control and limit the Cognitive Complexity of situations.

\section{Mental Models}

Mental models have been defined by Rouse and Morris (as cited in [8]) as "mechanisms whereby humans are able to generate descriptions of system purpose and form, explanations of system functioning and observed system states, and predictions of future states." Mogford has suggested there are two components of a controller's mental model: 1) a domain model of the airspace, aircraft, and required procedures for that airspace; and 2) a model of the devices used for surveillance and command implementation (i.e. the equipment used to perform the ATC task) [9].

Controllers use mental models as mechanisms to generate information to support their Situation Awareness [9]. A "working" mental model is a task-specific representation of the overall mental model. It is a mechanism used to support decision making processes at a level of abstraction appropriate for the current task.

\section{Abstractions}

Abstractions are a means of representing the essential characteristics of a mental model in a more cognitively compact form that is manageable within the constraints of human memory and processing limitations. Rasmussen states that the abstraction process is "not merely removal of details of information on physical or material properties. More fundamentally, information is added on higher level principles governing the co-function of the various functions or elements at the lower levels [10]."

Abstractions are powerful because they capture the most important and relevant aspects of a system without including the overhead of less critical details. This is hypothesized to reduce the Cognitive Complexity of performing that task, allowing the controller to attend to other aspects of the system.

\section{Structure as a Basis for Simplifying Abstractions}

Structure appears to form the basis for abstractions used in the working mental model in many ATC situations [5]. Structural elements can limit the potential future states of an air traffic situation. For example, standard operating procedures define standard ingress and egress points from a sector. The effect of these constraints is to reduce the number of likely aircraft trajectories entering and leaving a sector. Experience of the recurring patterns of behavior created by the presence of the underlying structure allows the creation of abstractions that simplify the level of detail required in the working mental model used to generate projections or perform the key ATC tasks. By simplifying the working mental model, those abstractions reduce the Cognitive Complexity of managing the evolution of the air traffic situation.

\section{Observability of Cognitive Complexity}

In Figure 1, Cognitive Complexity and the concept of complexity reducing abstractions are shown as internal to the controller working mental model. Mogford has previously identified complexity as an internal construct that cannot be directly measured or observed [11]. However, Cognitive Complexity can be investigated indirectly through observations of the controller and the ATC system. 


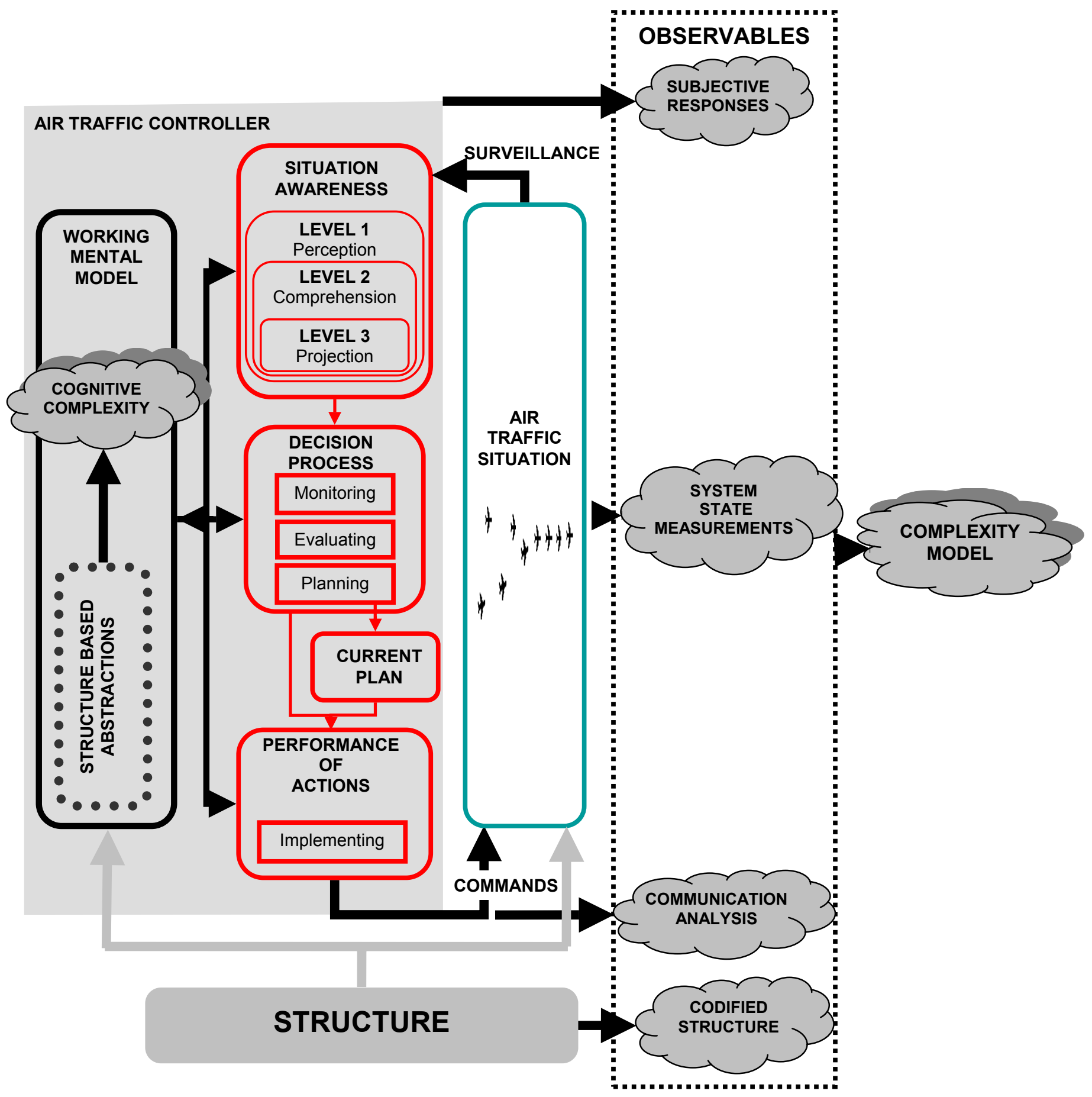

Figure 2. Observability Channels of the ATC system.

Figure 2 shows four observation channels that may be used to investigate the role of structure in reducing Cognitive Complexity. The subjective channel includes controllers' subjective responses to queries posed through interviews, questionnaires, or other subjective complexity assessment techniques. Such queries report controller's subjective sense of complexity, or "Perceived Complexity." However, a controller's perception can be influenced by many secondary factors which act as noise and can cause a divergence between the 
actual Cognitive Complexity and the perceived complexity.

A second observation channel is direct measurement of system states. These states can range from simple measures such as radar data to full aircraft states and trajectories as well as aggregate measures such as the number of aircraft in a sector or more complicated geometric properties.

A third observation channel is the commands and communication activity of the controller. Since this is a direct measurement of the controllers output it can be used to provide insight into the controllers' internal processes.

Finally, in ATC much of the structure is codified and can be observed through formal documentation, such as Standard Operating Procedures (SOPs), regulations, airways and airspace boundaries.

Because Cognitive Complexity is internal to the controller and is not directly measurable it is necessary to create a model which approximates the internal Cognitive Complexity. As shown in Figure 2 , the model is a conceptual framework that interprets observables and maps them into an external construct labeled as complexity. Importantly, because no direct measurement of Cognitive Complexity can be made, complexity models can only be validated against other observables or through hypothesis testing.

Complexity models can generate metrics used to predict internal Cognitive Complexity in operational settings or can be used to understand how system changes may impact Cognitive Complexity. An overly simple example of the application of such model is the number of aircraft in a sector, which forms the basis for the Monitor Alert function in the Enhanced Traffic Management System (ETMS) used to limit system capacity.

\section{Initial Observations}

Each of the four observable channels in Figure 2 can be used to probe how structure may act to reduce cognitive complexity. The following sections present initial examples of observations from each channel.

\section{Subjective Responses}

In order to determine the perceived complexity of an ATC situation, subjective responses can be elicited from controllers by a variety of mechanisms such as direct query, interviews, expert elicitation, etc (see [2] [4] [12] [13] [14]). However, as noted above, significant divergence between the perceived and Cognitive Complexity can occur. Therefore, the subjective data should be considered exploratory and should be correlated with objective measures whenever possible.

\section{Method}

In order to gather observations of controllers' perception of key Cognitive Complexity factors, a series of site visits to ATC facilities in the United States, Canada and France were conducted. The site visits included both en-route and terminal area control centers. The site visits consisted of focused interviews with current controllers, observations of live operations, as well as discussions with Traffic Management Unit and training personnel.

During the site visits, questions were posed that were designed to elicit a controller's perceptions regarding the use of structure. For example, controllers were asked to identify key factors which influence complexity. They were also asked to identify specific sectors or situations which they considered "easy" and "hard".

\section{Example Results}

Table 1 shows a list of the key factors controllers reported as influencing Cognitive Complexity. No attempt has been made to rank the factors. However, they have been found to fall into three categories: Airspace Factors, Traffic Factors, and Operational Constraints.

Airspace Factors are those related to properties of the airspace. Represented are both internal properties, such as the distribution of navigational aids, and external properties, such as sector shape and coordination activities. In general, these factors are quasi-static, characterizing the underlying context within which a traffic load exists.

Traffic Factors, are those dependent on the instantaneous distribution of traffic. They represent more dynamic and transient effects than Airspace Factors. Most previous efforts focused on measures associated with Traffic Factors. 
Finally, Operational Constraints are additional operational requirements that placed restrictions on possible control actions. These factors tend to represent short-term or temporary variations in operational conditions.

During the site visits to Boston Center controllers were asked to identify "easy" and "hard" sectors. The Albany sector was consistently identified as being harder than the Utica sector. Further analysis of the implications of these results is discussed in the following section.

\section{System State Measurements}

Direct observation of system states can be used to investigate the role and use of structure. Many of the previously proposed metrics of complexity have been based on such measurements including as the number of aircraft in a sector, the number of aircraft in transition (in altitude, speed, heading etc...), relative distances between aircraft, and sector transit time (see [1] [2] [4] [11]). Often these metrics are calibrated against observations of subjective data.

Observations of system states are appealing as they tend to be non-intrusive and require less data collection effort than subjective observations. However, they can also create a focus on only the geometrical relationships between aircraft which may miss some important structural factors that modify the Cognitive Complexity of a situation.

\section{Method}

System states can be determined from radar data and other state histories of aircraft together with logs of status states of other pertinent equipment such as radars, radios, weather etc... System state observations may also include flight plan data to capture presumed aircraft intent. In some cases observations of system states might also include aggregate or computed system properties [1].

To obtain insight into the existence of structure within the current ATC system, observations of traffic patterns have been made using data obtained from the Enhanced Traffic Management System (ETMS). Guided by findings from the subjective observations, this data was analyzed for evidence of key structural elements that could form the basis for structure based abstractions.
Table 1. Key factors reported by controllers as influencing complexity.

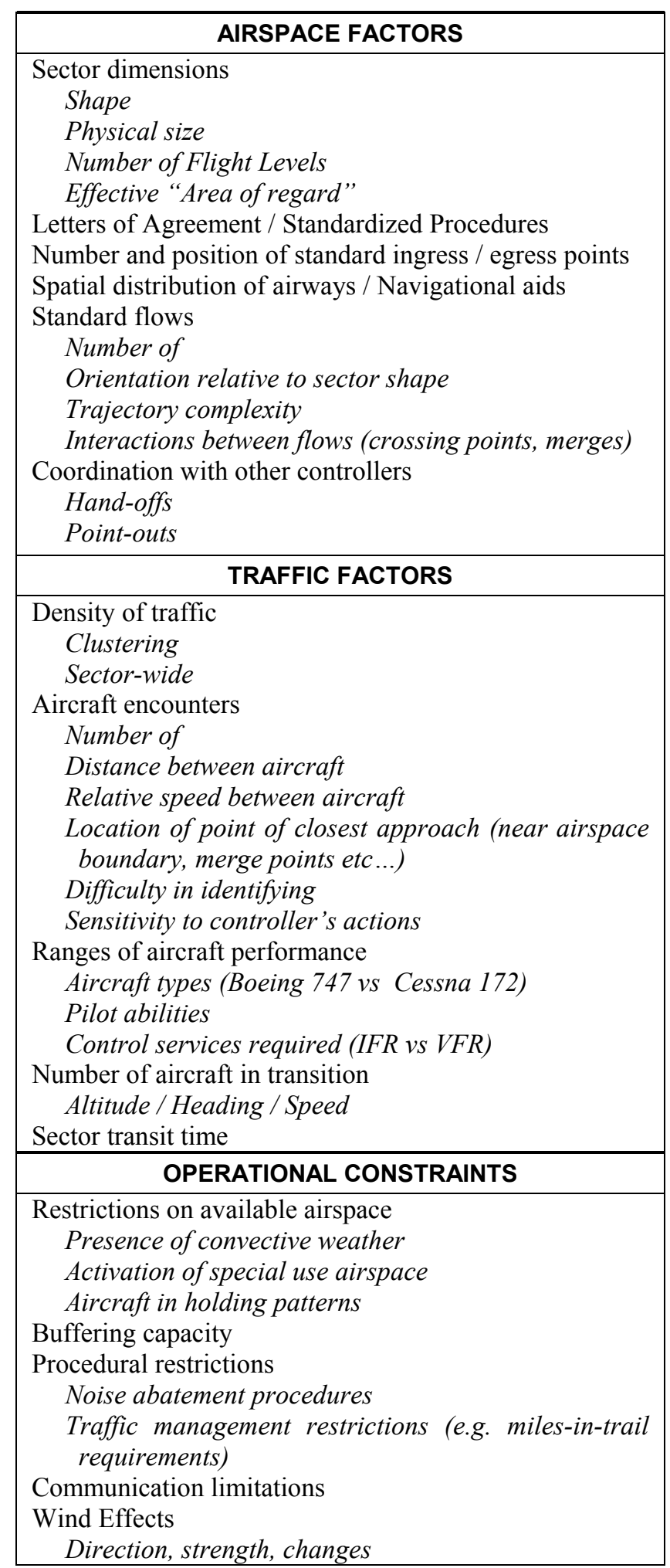




\section{Example Results}

Examples of observations using system state analysis of the "easier" (Utica) and "harder" (Albany) sectors as identified by Boston controllers are shown in Figure 3 and Figure 4. The figures show images of aircraft trajectories through the Utica and Albany sectors respectively. Approximately the same number of aircraft passed through each sector during the twenty four hour traffic sample (Utica: 268 aircraft; Albany: 231 aircraft).

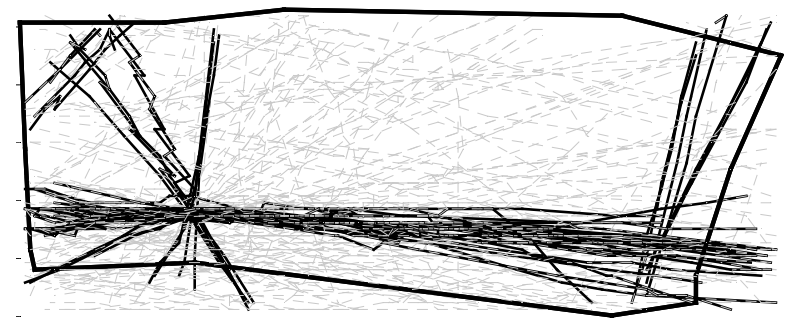

Figure 3. Image of 24 hours of traffic through Utica sector ("easier").

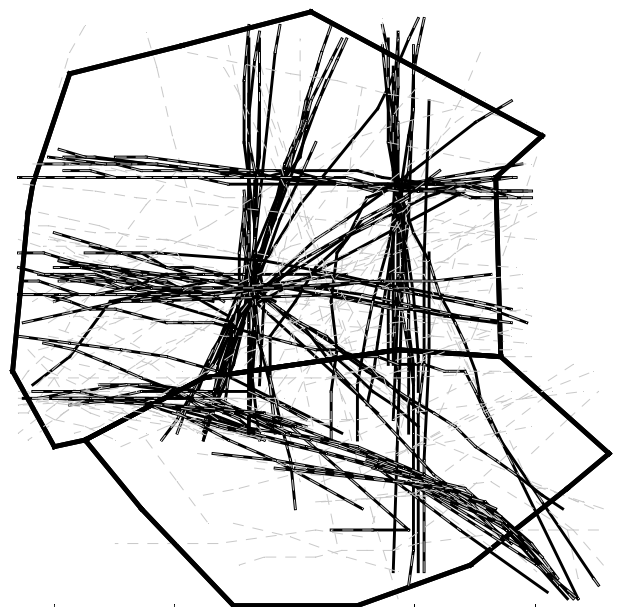

Figure 4. Image of 24 hours of traffic through Albany sector ("harder").

Analysis of the state data showed that there is more crossing traffic and a larger number of aircraft in vertical transition in the "hard" Albany sector than in the "easy" Utica sector (shown in Figure 3) which contained traffic flying predominantly in a uniform westbound direction.

In the subjective data controllers indicated the importance of the standard flows through a sector. The trajectories of aircraft that appeared to be on standard flows were identified and are highlighted by thick lines in Figures 3 and 4. As shown in
Figure 4 the "hard" Albany sector has more standard ingress and egress points, more standard flows and a greater amount of interaction between those flows than the Utica sector.

It is interesting to note that a higher fraction of the traffic is on identified standard routes in the "hard" Albany Sector $(\sim 43 \%)$ than in the Utica Sector $(\sim 20 \%)$. It appears that the controllers rely more heavily on the structured routes in the sectors they perceive as "hard" but are willing to allow more flexibility in situations which they consider "easy" This is consistent with subjective observations that the standard route abstraction is useful for reducing Cognitive Complexity. It also points out the fact that the controllers are active participants in managing their own cognitive complexity and will manage the system to maintain a safe buffer between their perceived complexity and their internally defined complexity limits.

System state data can also be used to identify important structural patterns at larger scales. Figure 5 shows an example of aircraft trajectories into Chicago O'Hare airport. The aircraft are clearly consolidated into flows that merge through a branching structure. Similar patterns have been observed at all major U.S. hubs.

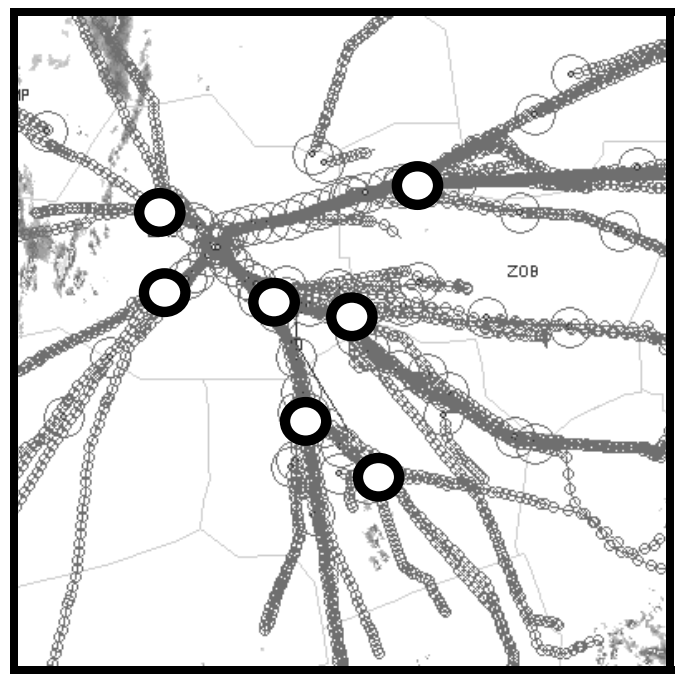

Figure 5. Standard Flows of Aircraft destined for O'Hare airport in Chicago.

As noted above controllers often rely on an abstraction based on an aircraft's membership in a standard flow. This abstraction is a key element in managing high density traffic. By classifying 
aircraft as members of a standard flow the aircraft dynamics are evaluated at the trajectory flow level and the stream is managed rather than the individual aircraft, thereby reducing the Cognitive Complexity per aircraft.

In order to generate the high density flows into the key airports it is necessary to merge the traffic into the 3 or 4 major flows which are typical of a hub airport. As can be seen in Figure 5 these mergers take place as defined "critical points" which are typically limited to a single critical point per flow per sector. It is hypothesized that there is a critical point abstraction which allows controllers to focus on critical merging and crossing points within their sector. In the case of a merge point, a critical point abstraction can reduce a multi-dimensional interaction problem to a one dimensional one of "time-of-arrival" at the merge point as the key control parameter.

\section{Codified Structure}

In ATC much of the structure is formally codified and documented. The explicitly documented structural elements used in conjunction with other channels to identify possible sources of structure based abstractions.

Codified elements of structure are not necessarily static objects reflecting airspace design. They include procedures and dynamic elements such as traffic management restrictions (e.g. MilesIn-Trail).

\section{Method}

Codified structural elements are identified through system documentation including navigational and airspace charts, standard operating procedures, letters of agreement, the controllers handbook and training material.

\section{Example Results:}

Figure 6 shows an example of structure in the MACEY TWO Standard Terminal Arrival Route (STAR) for Atlanta Hartsfield which delineates a common path and sequence of transitions that structure the flow of aircraft into the North-East corner post into Atlanta. When conjoined with other observation channels, the explicitly codified structure can provide valuable insight into the influence of structure on Cognitive Complexity.

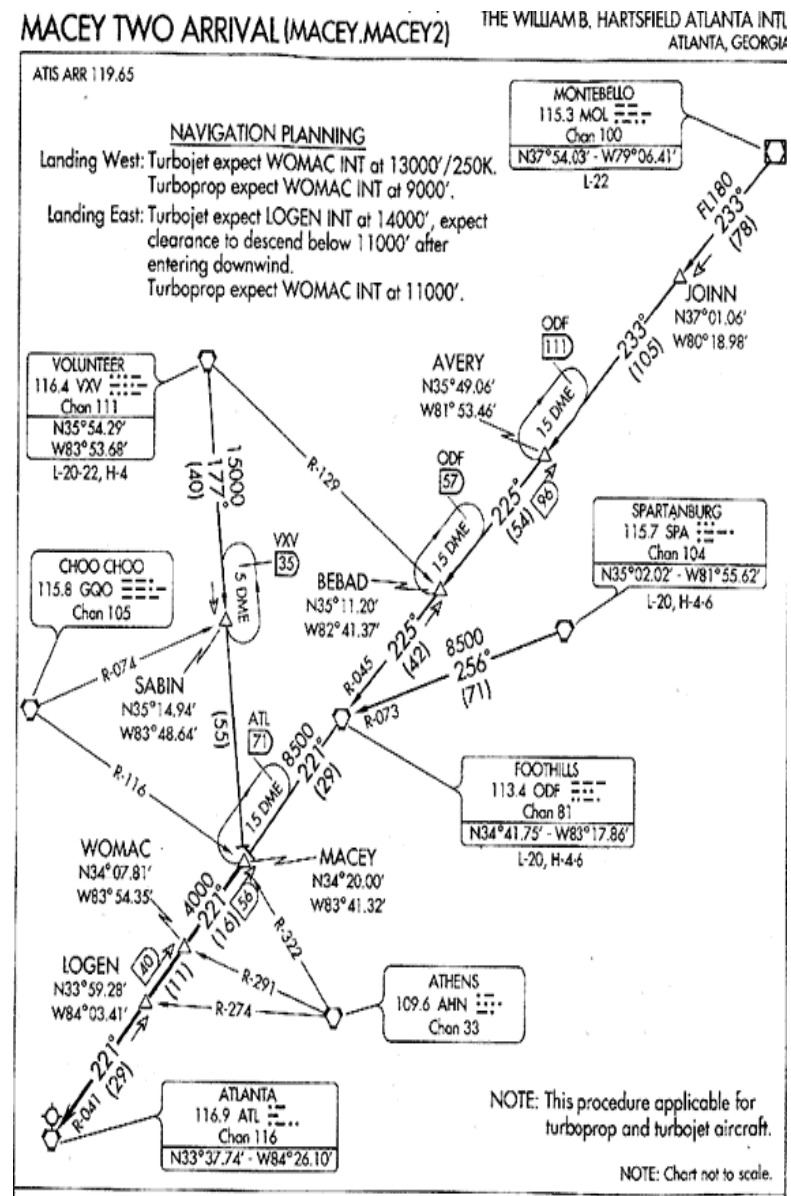

Figure 6. MACEY TWO Standard Terminal Arrival Route (STAR) (Courtesy [15]).

\section{Communication Analysis}

An additional source of observational data is the direct outputs from a controller in the form of commands and coordination activities which can be observed through the various forms of ATC communication. Previous investigations of ATC communications have generally focused on the duration of and frequency of verbal communication events [12]. However, additional insight into the controllers processes and the use of structure can be gained by more detailed analysis of the content of the controllers command output.

\section{Method}

In order to investigate the use of detailed communication analysis to gain insight into controllers decision processes and Cognitive Complexity a sector focused study was conducted of the Logen Sector of the Atlanta ARTCC. Controller-Pilot verbal communications were 
recorded [15] and correlated with ETMS data and weather data. Figure 7 depicts an example of the tracks through the sector over a 24 hour period. During this period $61 \%$ of all aircraft through the sector were destined for Atlanta-Hartsfield airport.

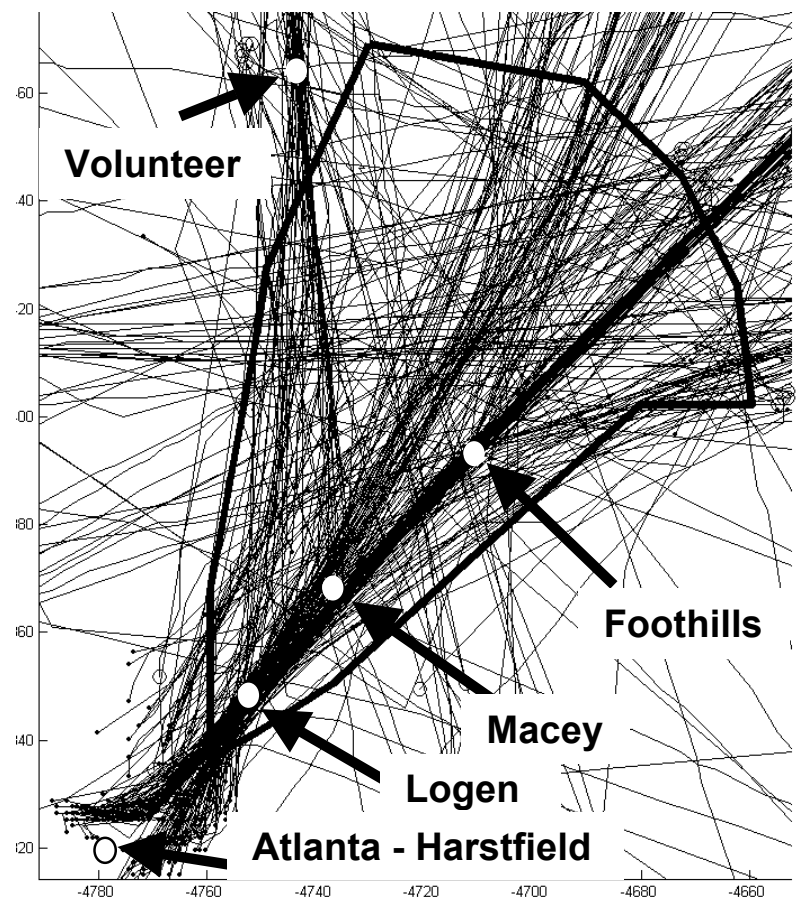

Figure 7. 24 Exposure of traffic flows through the Logen sector (Oct. 18, 2001).

The detailed analysis consisted of classifying communication events using a software application developed in Microsoft Visual Basic for this purpose which allowed the verbal data to be categorized and classified while the analysts could simultaneously visualize the traffic and weather situation. A screenshot of the communications analysis tool is shown in Figure 8.

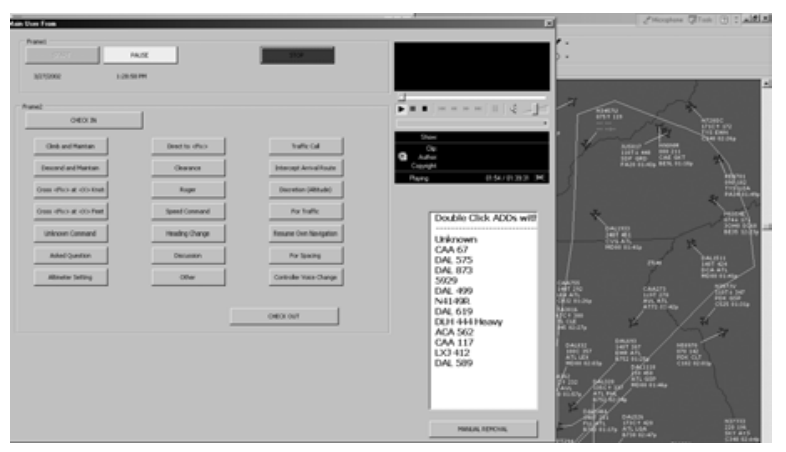

Figure 8. Screenshot of communications analysis tool (left) and visual display of air traffic situation based on ETMS data (right).
Based on a preliminary sample of the audio data, a classification scheme was developed which focused on controller-pilot communications and in particular the controllers commands which are considered the output of the controllers control process. It should be noted that pilot-controller communications were not analyzed. The command classification scheme reflected the commands observed in this sector, but is easily adapted for other sectors or applications.

\section{Example Results}

In order to illustrate how observations of commands could be used to investigate cognitive processes, a small pilot study was performed using 2.25 hours of audio and flight path data collected on Monday March 11, 2002. Initial analysis of the controller-pilot communication events showed that $47 \%$ of the events were commands.

The detailed analysis shown in Figure 9 indicates that three basic command types accounted for $61 \%$ of all issued commands over the observed period. When the commands were analyzed in the context of the codified structure of this sector (represented by the MACEY TWO STAR in Figure 6) it was found that a high percentage of the issued commands were consistent with the controllers executing the codified procedure. For example the standard flows through the sector were observed to converge and merge at the LOGEN navigation fix and $89 \%$ of the altitude crossing restrictions were issued for LOGEN which is the defined procedure for Atlanta arrivals for eastbound landings which was the condition during the observed period.

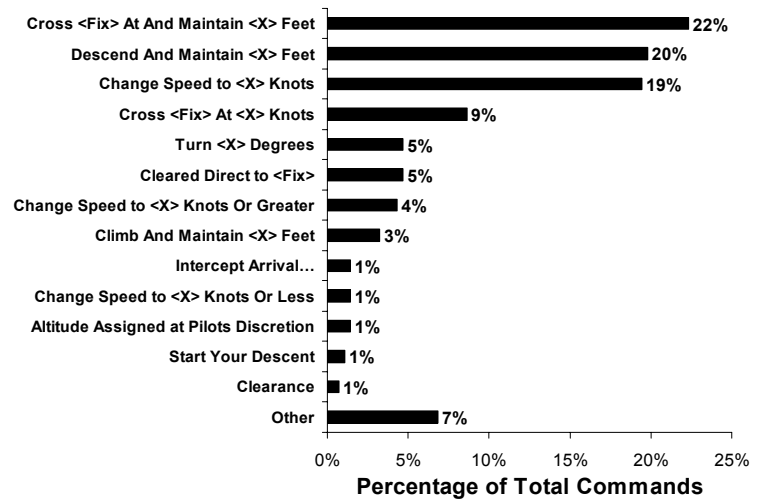

Figure 9. Relative occurrences of commands.

Additional insight into the importance of this codified structure for a controller's internal 
processes can be gained by investigating what happened when the underlying structural base was not available. In order to investigate this effect of loss of structure, the detailed communication analysis was conducted for periods where convective weather interfered with key portions of the MACEY TWO STAR. During June and July 2002, 1.5 hours of route disruption were observed, analyzed, and compared with 4 hours of observations in clear weather periods. The command analysis are shown in Figure 10 which compare the number of commands per aircraft for conditions of the LOGEN fix being clear and blocked.

When the key LOGEN fix was blocked, the number of crossing restrictions was substantially reduced, suggesting the controllers were no longer using the structure provided by the MACEY TWO STAR. In addition, and perhaps in reflection of the loss of capacity which occurred when the underlying structure was compromised, a strategic response of the system was observed. The traffic rates through the sector were reduced by approximately $1 / 3$ during the disrupted operations compared with the baseline operations. The mix of traffic was also shifted with the air carrier traffic being reduced from $71 \%$ in the nominal condition to $34 \%$ in the disrupted condition.

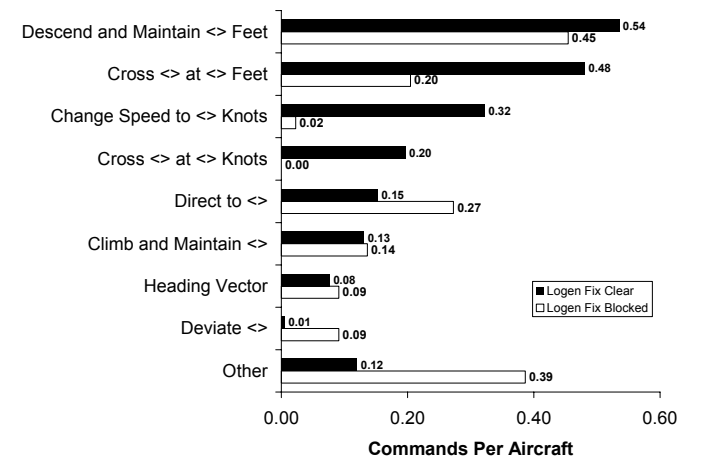

Figure 10. Commands per aircraft with the LOGEN fix blocked and clear of convective weather.

\section{Experimental Probes of Structure Based Abstractions}

One approach to validate "structure based abstractions" is to subject the abstractions to experimental validation. If a structure based abstraction is hypothesized and the structural basis is manipulated as an independent variable then, according to the model in Figure 2, the subjective data (perceived complexity), objective data (system states and performance) and the command data (ATC commands) should reflect the change in perceived complexity. This approach was tested using the "critical points" abstraction discussed in the section System State measurements. The experiment was conducted in order to evaluate the hypothesis that a single co-located merge point was an effective structural abstraction.

\section{Method}

A simplified Air Traffic Control Task was simulated where subjects attempted to merge streams of traffic using only speed commands to achieve a minimum in trail separation upon leaving the sector. An example of the simulation display is shown in Figure 11. The independent variables in the experiment were the number of incoming traffic streams and if the streams merged at a single "colocated" merge point or if each stream merged separately. The test matrix is shown in Table 2.

The dependant variables were the number of separation violations, number and magnitude of command and subjective ratings of the difficulty (using a modified Cooper-Harper rating scale). The incoming traffic streams were constructed of modular segments which were rotated for each test case to provide identical load statistics with an unpredictable pattern to the controller.

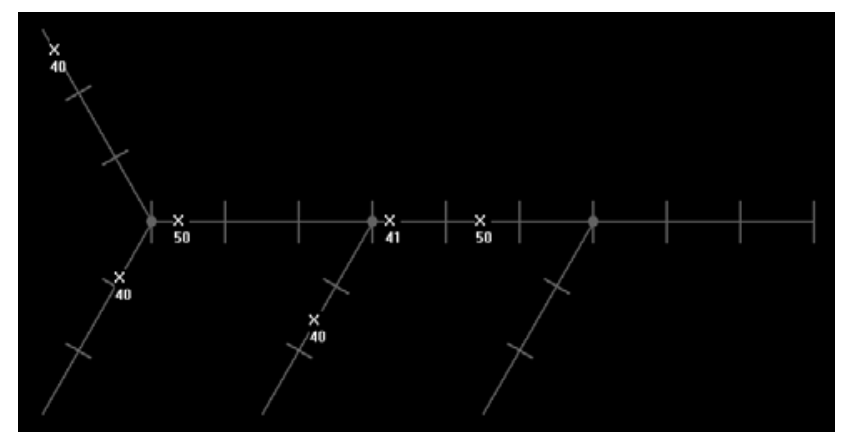

Figure 11. Screenshot of ATC simulation environment. Numbers indicate the speed of the aircraft. 
Table 2. Merge point arrangements used in experimental study of critical point abstraction.

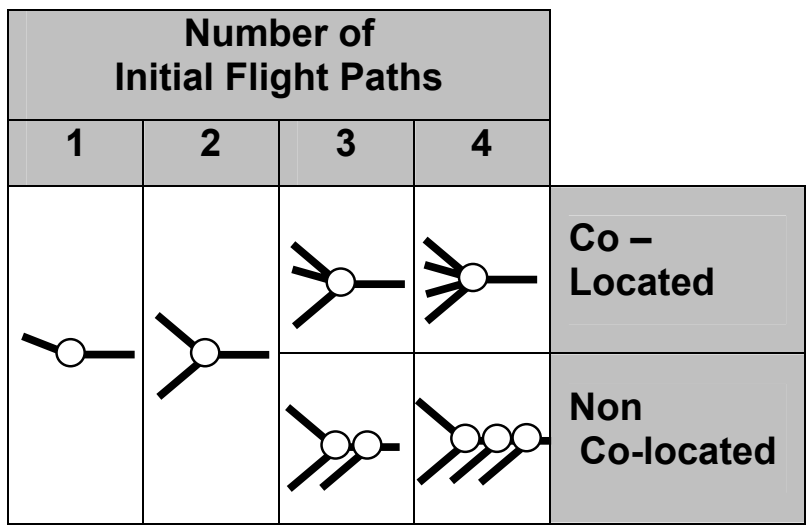

\section{Results}

In the initial study, 12 university students participated as controllers. Initial results support the hypothesis that a co-located merge point simplifies the merging task. The average number of separation violations is shown in Figure 12. The average number of violations was less than 1 for the colocated merge points and approximately 2.3 for the non-collocated merge points. Due to the relatively small number of violations these results are only significant at the $90 \%$ confidence level but are consistent with the underlying hypothesis and the other observational data. It is interesting to note that close to $90 \%$ of all separation violations which occurred in the non co-located scenarios involved an aircraft joining the main horizontal flow at the second or third merge point.

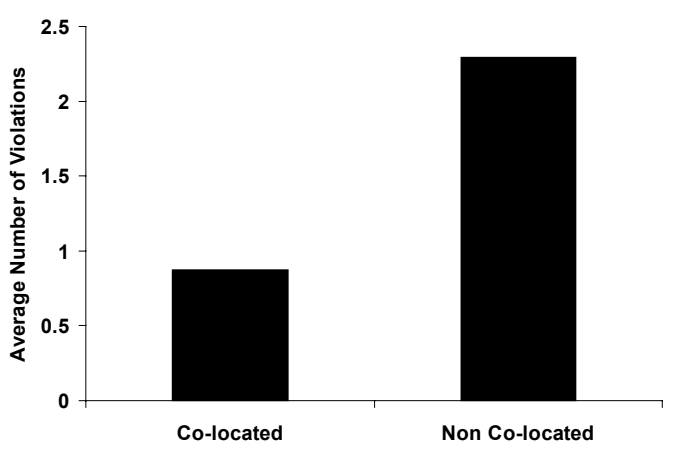

Figure 12. Separation Violations.

As seen in Figure 13 the amount of commanded speed change was higher for the non co-located merge points. This result is statically significant and is consistent with the hypothesis that the abstractions based on the co-located merge points simplified the controller tasks presumably through a less complex mental model.

The subjective Cooper-Harper ratings of difficulty in controlling the situation were also consistent with the hypothesis. As seen in Figure 14 subjects perceived the co-located scenarios as less difficult than the non co-located scenarios.

A more detailed description of the experiment can be found in [16] and [17] but the results suggest that "structure based abstractions" can be successfully probed through indirect experimental techniques.

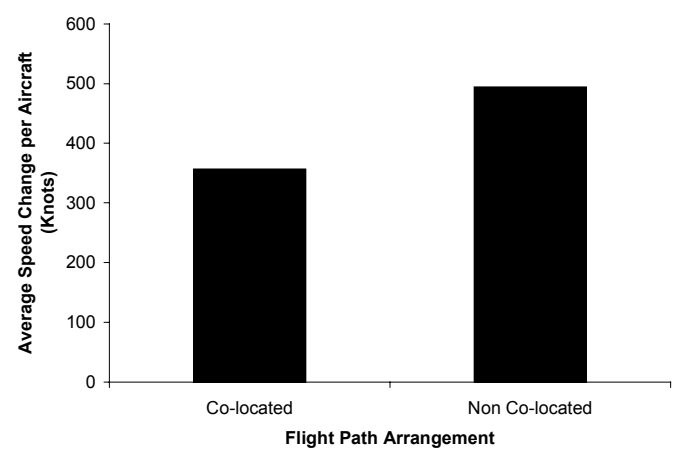

Figure 13. Average Speed Change Commanded per Aircraft.

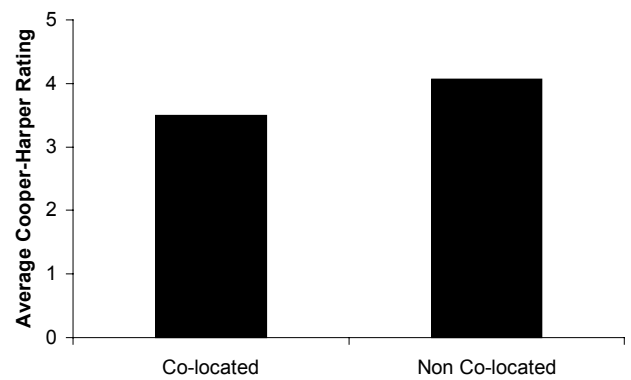

Figure 14. Cooper Harper ratings.

\section{Conclusions / Summary}

In order to develop an understanding of the impact of structure on the controllers internal Cognitive Complexity, a broad observational and holistic approach has been proposed to overcome the lack of direct observability of controllers internal processes. Based on this approach, some 
initial examples of structure-based abstractions (e.g. standard flows and critical points) have been identified and supported by experimental and observational probes. The underlying structural abstractions which have been investigated clearly act to reduce the Cognitive Complexity of the controllers working mental model and allow the capacity of the ATC system to be higher than when the structure based abstractions are not available. The structure may sufficiently reduce controllers cognitive load, to allow more flexibility for aircraft in the system. While the results are preliminary the multi-channel approach appears to be a promising method to investigate the impact of structure on controllers Cognitive Complexity and it's impact on ATC operations.

\section{Acknowledgements}

The authors would like to thank the air traffic controllers who shared their experiences and provided opportunities for observation. The MIT work was supported by FAA grant \# 96-C-001 through the National Center of Excellence for Aviation Operations Research (NEXTOR), Agreement \# SA 1603JB. The CENA work was supported by CENA, ENAC, and LAAS. The image in Figure 5 was captured using "Flight Explorer," a commercial product providing an ETMS data feed. The authors also acknowledge Hayley Davison for her contributions and perspectives on the concept of abstractions and mental models.

\section{References}

[1] Delahaye, D., S. Puechmorel, 2000, Air Traffic Complexity: Towards Intrinsic Metrics, Napoli, 3rd USA/Europe Air Traffic Management R\&D Seminar.

[2] Laudeman, I., S.G. Shelden, R. Branstrom, C.L. Brasil, 1998, Dynamic Density: An Air Traffic Management Metric, NASA-TM1998-112226.

[3] Sridhar, B., K.S. Seth, S. Grabbe, 1998, Airspace Complexity and its Application in Air Traffic Management, Orlando, 2nd USA/Europe Air Traffic Management R\&D Seminar.
[4] Wyndemere Inc., 1996 An Evaluation of Air Traffic Control Complexity, Final Report NASA 2-14284.

[5] Histon, J.M., Guillaume Aigoin, Daniel Delahaye, R.John. Hansman, Stephane Puechmorel, 2002, Introducing Structural Considerations into Complexity Metrics, Volume 10, \#2, Air Traffic Control Quarterly.

[6] Endsley, M, M. Rodgers, 1994, Situational Awareness Requirements for En-route Air Traffic Control, DOT/FAA/AM-94/27.

[7] Pawlak, William S., Christopher R. Brinton, Kimberly Crouch, Kenneth M. Lancaster, 1996, A Framework for the Evaulation of Air Traffic Control Complexity, San Diego, Proceedings of the AIAA Guidance Navigation and Control Conference.

[8] Endsley, M., 2000, Situation Models: An Avenue to the Modeling of Mental Models, 44th Annual Meeting of the Human Factors and Ergonomics Society.

[9] Mogford, Richard H., 1997, Mental Models and Situation Awareness in Air Traffic Control, Volume 7, \#4, International Journal of Aviation Psychology.

[10] Rasmussen, J. 1986, Information Processing and human-Machine Interaction: An Approach to Cognitive Engineering, Amsterdam, Elsevier Science Publishing Co. Inc.

[11] Mogford, Richard H, J.A. Guttman, S.L. Morrow, P. Kopardekar, 1995, The Complexity Construct in Air Traffic Control: A Review and Synthesis of the Literature, U.S. Department of Transportation FAA Report Number DOT/FAA/CT-TN92/22.

[12] Manning, Carol, Scott Mills, Cynthia Fox, Elaine Pfleiderer, Henry Mogilka, 2001, The Relationship Between Air Traffic Control Communication Events and Measures of Controller Taskload and Workload, Sante Fe, 4th USA/Europe Air Traffic Management R\&D Seminar.

[13] Mogford, Richard H., E.D. Murphy, J.A. Guttman, 1993, Using Knowledge Exploration Tools to Study Airspace 
Complexity in Air Traffic Control, Volume 4, \#1, The International Journal of Aviation Psychology.

[14] Mogford, Richard H., E.D. Murphy, R.J. Roske-Hofstrand, G. Yastrop, and J.A. Guttman, 1994, Research Techniques for Documenting Cognitive processes in Air Traffic Control: Sector Complexity and Decision Making, U.S. Department of Transportation FAA Report Number DOT/FAA/CT-TN94/3.

[15] www.atcmonitor.com

[16] Gottlieb, Blake, 2002, Testing Air Traffic Controllers' Cognitive Complexity Limits, Class Project Final Report.

[17] Kleinwaks, Howard, 2002, Testing Air Traffic Controllers' Cognitive Complexity Limits, Class Project Final Report. 